\title{
The Application and Experience by Professor Xia Guicheng's Theory of Tonifying Kidney and Regulating Menstruation on Female Sub-Health
}

\author{
Wan Zhaoxia', Shi Yaping ${ }^{1}$, Fan Yichen ${ }^{2}$ \\ ${ }^{1}$ Gynecology Department, Nantong Hospital of Traditional Chinese Medical, Nantong, China \\ ${ }^{2}$ Basic Medical School, Nanjing University of Chinese Medicine, Nanjing, China
}

Email address:

wzx4394@126.com (Wan Zhaoxia)

\section{To cite this article:}

Wan Zhaoxia, Shi Yaping, Fan Yichen. The Application and Experience by Professor Xia Guicheng's Theory of Tonifying Kidney and Regulating Menstruation on Female Sub-Health. European Journal of Preventive Medicine. Vol. 6, No. 3, 2018, pp. 34-37. doi: $10.11648 /$ j.ejpm.20180603.11

Received: June 18, 2018; Accepted: July 17, 2018; Published: August 9, 2018

\begin{abstract}
Objective: to explore the possible mechanism of sub-health in women from the perspective of traditional Chinese medicine, and to explore the mechanism of treating sub-health status in women at different physiological stages by using professor xia guicheng, a master of traditional Chinese medicine, his theory of tonifying kidney and regulating menstruation. Methods: through the sharing and interpretation of clinical cases of female sub-health, the occurrence mechanism, therapeutic effect and action mechanism were analyzed. Conclusion: the method of tonifying kidney and regulating menstruation by professor xia guicheng, can effectively solve the sub-health status of women.
\end{abstract}

Keywords: Female Sub-health, Xia Guicheng, Tonifying Kidney and Regulating Menstruation, Experience

\section{Introduction}

Sub-health refers to a state in which there is no obvious disease, but the activity is decreased and the adaptability is decreased to varying degrees. Because of the special status and special role of women in society and family, their health status is paid close attention to, and their sub-health status is relatively prominent, which needs the help of the society, as well as the help of doctors $[1,10]$.

Female sub-health is a dynamic process, it is either to the state of disease (Including Chinese medicine, acupuncture, massage and so on) [14-16], or to the state of health $[2,3]$.

$\mathrm{Xia}$ Guicheng is a famous Chinese medicine gynecologist, master of Chinese medicine, chief physician of gynecology of Jiangsu Provincial traditional Chinese Medicine Hospital, professor, master's degree, doctoral supervisor, and enjoys special allowance from the government. In TCM gynecology major engaged in medical treatment, teaching, scientific research for 60 years, on the basis of a profound understanding of the changes in the menstrual cycle of women, combined with the corresponding view of heaven and man, the theory of "heart, kidney and uterus axis" was founded. The establishment of menstrual cycle rhythm (we are used to call it the theory of tonifying the kidney and regulating the menstruation) has become the fundamental method for the treatment of female diseases [4-6].

Inspired by this, we apply Professor Xia Guicheng undefineds theory to adjust female sub-health state and obtain good results. Through different medical records analysis, talk about clinical experience.

\section{Typical Case}

\subsection{Case NO.1 Adolescence Is Not Focused Enough}

\subsubsection{Treatment Process}

Fan 17, senior high school student. Initial consultation time: 2011-3-4. Due to the lack of attention in the premenstrua for 3 months. Last menstrual period: 2011-2-28. Menophania when she was 14 , menstrual cycle rules, $5 / 30$ days, menstrual volume medium, with occasionally painful, tolerable. Main complaint: Every time in the premenstrua, attention is not focused enough, affecting the learning effect. Accompanied performance: sleep poor, bitter mouth, occasionally anxiety, 
lumbar acid, normal urine, dry stool, once a day. The tongue is red, the Moss is thin, and the veins are slightly and weak. Laboratory tests and other tests: sex hormones, thyroid, electrolytes, and B ultrasound did not indicate abnormalities. At the time of delayed menorrhea, I was given the method of nourishing kidney, nourishing Yin, and promoting Qi and sparse liver. Selecting the prescription of Professor Xia Guicheng's method of tonifying the Kidney and regulating the week of later stage additions and subtractions:Danshen $10 \mathrm{~g}$, Baishao 10g, Huai Shanyao 15g, Shan Zhuyu 10g, Fuling 10g, Tu Sizi 15g, He Huanpi 10g, Lian Zixin 3g, Zhi Huangqi 20g, Dangshen 10g, Baizhu 10g, Chaihu 6g, Longdan 10g 7 dose Non-fried granules, one dose per day, twice a day (the same method below). Also give psychological counseling and ask her parents not to exert too much pressure. Second consultation: 2011-3-11. Main complaint: a little white belt like egg white, one week later will be insufficient attention, accompanied by breast swelling pain, occasional anxiety, bitter mouth seems to improve, good or bad time when sleeping. The tongue is red, the moss is thin, and the veins are slightly and weak. At the time of the transition from the interim period to the early period, it is proposed to supplement the kidney to help Yang and Yi Qi to sparse the liver. Selecting the prescription of Professor Xia Guicheng's method of tonifying the Kidney and regulating the week of former stage additions and subtractions:Danshen 10g, Zhi Huangqi 20g, Baishao 10g, Huai Shanyao 15g, Yin Yanghuo 10g, Chuanduan 15g, Duzhong 10g, Tu Sizi 15g, Zi Shiying 10g, Danshen 10g, Fuling 10g, Chaihu 6g, Jingjie 10g, Lu Jiaoshuang 10g, 14 dose. Non-fried granules, one dose per day, twice a day. Three consultations: 2011-3-26. Main complaint: a little bleed from vagina, lower abdomen swelling, breast swelling than the previous tolerance, emotional stability, light tongue, thin moss, veins and strings and slippery. During menstrual period, it is proposed to supplement the activating blood to promote menstruation and soothing the liver. Selecting the prescription of Professor Xia Guicheng's method of tonifying the Kidney and regulating the week of menstrual period additions and subtractions: Danggui $10 \mathrm{~g}$, Chuan Niuxi $10 \mathrm{~g}$, Chishao $10 \mathrm{~g}$, Fuling 10g, Qinpi 6g, Chenpi 6g, Yan Husuo 10g, Moyao 6g, Chaihu 6g, Jingjie 10g, 7 dose. Non-fried granules, one dose per day, twice a day. In this way, two menstrual cycles will be taken again. After 3 months, the mother informed that all aspects are right, and the learning efficiency has increased and the mood was stable.

\subsubsection{Discussion}

Adolescent female sub-health due to the heavy burden of academic stress, too little exercise and lack of sleep, fatigue, anorexia, bitter mouth, anxiety, pain in premenstrual breasts, dysmenorrhea, and so on. Generally, it can be alleviated by psychological guidance and proper rest. However, some children need traditional Chinese medicine to help solve the problem. In combination with the theory of the fourth period of menstruation, attention is paid to the use of spleen and liver care to improve the effect $[7,8]$.

\subsection{Case NO.2 Emotions of Childbearing Age}

\subsubsection{Treatment Process}

Chen, 36, bank clerk. Initial consultation time: 2013-7-11. Appeal: Because of frequent feeling tired, emotional depression for two months. Last period: 2013-5-27. Menophania when she was 13 , the previous menstrual cycle rules, 5-7 / $30 \pm 2$ days, menstrual volume medium, occasional dysmenorrhea, forbearance, fertility history: 1-0-0-1 / 10 years old, cesarean section, contraceptive methods. In the past two months, she often felt tired and depressed. Menstruation is still regular, 23-35 days. Accompanied performance:, diet normal, sleep general, occasionally bitter mouth, impatient, occasional lumbar acid, normal urine, dry stool, two days have stool. Tongue red, Moss thin, pulse string. Laboratory examinations and other: sex hormones, thyroid, electrolytes did not indicate abnormalities, B ultrasound showed normal uterine size morphology, inner membrane $1.2 \mathrm{~cm}$, bilateral ovary normal size. During the early period, it is proposed to supplement the activating blood to promote menstruation and soothing the liver. Selecting the prescription of Professor Xia Guicheng's method of tonifying the Kidney and regulating the week of menstrual period additions and subtractions: Danggui 10g, Chuan Niuxi 10g, Chishao 10g, Fuling 10g, Taoren 10g, Honghua 6g, Chaihu 6g, Long Dan 10g, Baizhu 20g, Rou Congrong 10g, 7 dose. Non-fried granules, one dose per day, twice a day. Second consultation: 2013-7-20. Appeal: After the medicine, she was menstruated the next day, passing thoroughly, and the amount was available. Occasionally, there was lumbar acid, and the other of the main complaints were unchanged. The tongue was red, the moss was thin, and the pulse strings were slightly fine. After the later period, I was given the method of nourishing kidney, nourishing Yin, and promoting Qi and sparse liver. Selecting the prescription of Professor Xia Guicheng's method of tonifying the Kidney and regulating the week of later stage additions and subtractions: Tu Sizi 10g Shan Zhuyu 10g, Sheng Dihuang 10g, Huangbai 6g, Zhi Huangqi 20g, Dangshen 10g, Zexie 10g, Fuling 10g, Baizhu 10g, Jue Mingzi 10g, 10g, Chaihu 6g, Longdan 10g 7 dose. Non-fried granules, one dose per day, twice a day At the same time, give psychological comfort and tell her not to be overly nervous. Three consultation: 2013-7-27. The appearance of white belt like egg white, mouth pain turn right, mood is still stable, good time difference when sleeping, stool improved. The tongue is red, the moss is thin, and the veins are smooth. At the time of the transition from the interim period to the early period, it is proposed to supplement the kidney to help Yang and Yi Qi to sparse the liver. Selecting the prescription of Professor Xia Guicheng's method of tonifying the Kidney and regulating the week of ovulation promotion additions and subtractions: Danggui $10 \mathrm{~g}$, Danshen $10 \mathrm{~g}$, Baishao 10g, Chishao 10g, Chuanxiong 6g, Danpi 10g, Fuling 10g, Honghua 6g, Zelan $10 \mathrm{~g}$, Chaihu $6 \mathrm{~g}$, Jingjie $10 \mathrm{~g}$, Roucongrong $10 \mathrm{~g}, 5$ dose. Non-fried granules, one dose per day, twice a day. Four consultation: 2013-8-3. Appeal: slight breast swelling, lumbago, slight mouth pain, normal bowel movements. The tongue is red, the moss is thin, and the veins 
are smooth. It is in the later period of the early period. it is proposed to supplement the kidney to help Yang and to sparse the liver., Selecting the prescription of Professor Xia Guicheng's method of tonifying the Kidney and regulating the week of early period $\mathrm{Cu}$ Haungtang additions and subtractions: Dangshen 10g, Zhi Haungqi 20g, Ji Xueteng 30g, Xiangfu 10g, Xianmao 10g, Xian Lingpi 20g, Zeland 10g, Zexie 10g, Che Qianzi 15g, Tian Huafen 20g, Chaihu 6g, Longdan 6g, Lu Jiaoshuang 10g, 14dose. Non-fried granules, one dose per day, twice a day. Five consultations: 2013-8-17. Appeal: The lower abdomen is swollen, double breast are swollen, the tongue is light, the moss is thin, and the veins are smooth. Passing the precursor, intended to move blood and through the scriptures, with sparse the liver as the method, the initial diagnosis of the party to subtract Chaihu, Longdan, 7 doses. Non-fried granules, one dose per day, twice a day. Six consultations: 2013-8-25. Appeal: The energy and spirit were greatly improved compared to the previous one, and the sense of fatigue disappeared. In the previous month. Consultations not repeated.

\subsubsection{Discussion}

Sub-health status of women in reproductive age is more common, but also a trend of multiple. Because women in this stage bear many changes in the family, society, and their own physiological cycles, this part of the women, despite the absence of many so - called disease - positive manifestations, they are enough for doctors to pay attention and can not be ignored, Because strictly speaking, this group of women is already on the verge of getting sick. so as doctors, be active with them in order to prevent the disease from happening. Some studies have suggested that the sub-health status of women at this stage is closely related to the liver. In practice, the common symptom groups of such sub-healthy women such as emotional anxiety, depression, irritability, sleep disorders and other chronic psychological stress symptoms, as well as mild or abnormal menstruation, are mostly signs of liver loss, leakage, liver depression, and stagnation. Therefore, during the adjustment of the week, emphasis was placed on liver depression $[9,10]$.

\subsection{Casa NO.3 Poor Sleep During Peri-menopausal}

\subsubsection{Treatment Process}

Lee, 45 years old. Initial consultation time: 2014-5-4. Due to poor sleep, mood swings, and occasional night sweats for three weeks. Last menses: 2014-4-17. Menophania at 15 years old, menstrual cycle rules, 5/30 days, Menstrual volume medium, occasional dysmenorrhea, forbearance, childbearing history: 1-0-2-1 / 19 years old, natural delivery. Use of birth control ring. Accompanied symptoms: Normal diet, when there is mouth pain, and dry, occasional lumbar acid, normal urine, stool slightly embarrassed, once a day. The tongue is light, the Moss is thin and white, and the veins are slightly and weak. Laboratory tests and others: The third day of menstruation showed FSH12.5mIU/L, E221pg/L, no significant abnormalities, and thyroid, electrolyte, and B ultrasound tests did not indicate abnormalities. Come to ask for conditioning. The menstrual cycle of the patient is still regular, and it is engraved at the beginning of the menstrual period. Therefore, it is planned to supplement the kidney to help Yang, Yi Qi to strengthen the spleen, and clear the heart and sparse liver. Selecting the prescription of Professor Xia Guicheng's method of tonifying the Kidney and regulating the week of early stage additions and subtractions: Zhi Huangqi 20g, Fuling 10g, Jiang Banxia 6g, Qingpi 6g, Ji Xueteng 30g, Xiangfu 10g, Xianmao 10g, Xian Lingpi 20g, Zelan $10 \mathrm{~g}$, Zexie 10g, Che Qianzi 15g, Gouteng 10g, Chaihu 6g, Longdan 6g, Ma Huanggen 20g, 14 dose. No fried granules, one dose per day, twice a day. Second consultation: 2013-6-1. Comfortable after the medicine, night sweat relief, sleep is still poor, stool normal. The tongue is light purple, the moss is thin, and the veins are smooth. At the time of the passage, it is planned to move blood through the scriptures, with Yiqi Jianpi andclear the heart and sparse liver as the method. Selecting the prescription of Professor Xia Guicheng's method of tonifying the Kidney and regulating the week of menstrual period additions and subtractions: Zhi Huangqi 20g, Danggui 10g, Chuan Niuxi $10 \mathrm{~g}$, Chishao $10 \mathrm{~g}$, Fuling $10 \mathrm{~g}$, Chenpi $6 \mathrm{~g}$, Sanleng 10g, Ezhu 10g, Gouteng 10g, Ye Jiaoteng 30g, He huanpi $15 \mathrm{~g}$, Chaihu $6 \mathrm{~g}$, Jingjie $10 \mathrm{~g}$, Ma Huanggen $10 \mathrm{~g}, 14$ dose. Non-fried granules, one dose per day, twice a day. Three visits: 2013-6-9. The patient's feeling situation has greatly improved, consciously comfortable, and requires consolidation. The tongue is light, the moss is thin, and the veins are slightly thin. At the end of the period, the method of nourishing the kidney, nourishing the spleen and sparse the liver was used. Selecting the prescription of Professor Xia Guicheng's method of tonifying the Kidney and regulating the week of later stage additions and subtractions: Tu Sizi $10 \mathrm{~g}$, Shan Zhuyu 6g, Sheng Dihuang 30g, Huangbai 6g, Tiandong 10g, Maidong 10g, Gouteng 10g, Zelan 10g, Fuling 10g, Zhi Huangqi 20g, Chenpi 6g, Chaihu 6g, Jingjie 10g, 7 dose. Non-fried granules, one dose per day, twice a day. After three months, he was told that he had stopped taking medication after one month because of no obvious discomfort. He hoped to help in similar cases in the future.

\subsubsection{Discussion}

Perimenopausal subhealth state is relatively few, but once appear, often more intense reaction. Because at this stage, due to special physiological reasons, ovarian function gradually declines, estrogen levels gradually and gradually decline, resulting in a series of discomfort. If they encounter pressure from family and work, their discomfort will be amplified. They need for attention, care, and care are relatively more, so we emphasize the methods of kidney supplementation, liver care, and clear away heartfire at the same time as clinical adjustment [11-13].

\section{Conclusion}

"The theory of regulating the period" is established by Professor Xiaguicheng, a master of Chinese medicine, according to the characteristics of female's physiological 
cycle in long-term clinical practice, and effectively guides clinical practice. So that many young doctors in the treatment of gynecological diseases deeply understand the advantages of Chinese medicine and consciously develop the advantages of Chinese medicine. Not only that, it also has guiding significance in the Chinese medicine activities where women are not ill or women are sub-healthy. In our long-term clinical practice, we have also learned that there are different sub-health priorities for women at different physiological stages of their lives. The combination of Professor Xiaguicheng's "theory of regulating the period" and the "dialectical treatment" of the soul of Chinese medicine and its flexible application to women in sub-healthy States at different physiological stages have achieved good results. It brings calm and confidence to the masses of sub-healthy women and can effectively prevent the occurrence and development of diseases.

Of course, appropriate psychological counseling, moderate exercise, massage and acupuncture interventions by traditional Chinese medicine are also effective means to solve female sub-health. Its main purpose is to improve the quality of life of female sub-healthy people and contribute to the harmony of the family and society.

\section{References}

[1] Wangwenzhi, Chenzhenghong Yanyuxiang and others. Research on sub-health status-Journal of Chinese General Practitioners2014/13(4): 286 288.

[2] Wangtianfang. Discussion on the Concept, Category and Interrelation of Subhealth and Healing Uncured Disease. 29(10): 929-933.

[3] Chinese Traditional Chinese Medicine Association. "Subhealth Traditional Chinese Medicine Clinical Guide"(ZYYXH/T2 -2006) Beijing: China Traditional Chinese Medicine Press, 2006: 1-2.

[4] Xiaguicheng and so on. Chinese Medicine Clinical Gynecology People's Health Press, 1994.
[5] Xiaguicheng and so on. "Diagnosis and treatment of Chinese medicine for menstrual diseases" People's Health Publishing House, 2001.

[6] Tanyong et al. Kun Hu Ying-Xiaguicheng's gynecological insight. People's Health Publishing House, 2014:20-24.

[7] Heqinghu, Zhou Xing. Women's sub-health is based on liver. China Traditional Chinese Medicine Modern Distance Education. 2008, 6(7): 707-708.

[8] Cen Rui Shen. A brief talk on the Strategy of Chinese Medicine to deal with Sub-health State [J]. Chinese Medical Forum 2003 18 (03): 44-45.

[9] Wei Hong, Zheng Hongxin. Discussion on Sub-health status and intervention measures of TCM [J]. Liaoning Journal of traditional Chinese Medicine, 200431 (5): 39-40.

[10] Liu Qiu Ling. A Survey of Sub-health in Professional Women [J]. Modern Preventive Medicine 200734 (8): 1525-1526.

[11] Liu Bao-yan, he Li-yun, Xie Yan-ming, et al. The development of questionnaire on basic syndromes of Chinese medicine in sub-health state $[\mathrm{J}]$. Chinese Journal of basic traditional Chinese Medicine 2004, 10(9): 23-28.

[12] Wang ce, Dong Sheng. Progress in TCM treatment of sub-health state [J]. Journal of Shaanxi Institute of traditional Chinese Medicine 2009, 32(2): 73-75.

[13] Yan Li, Liu Xingshan, Jiang Jie, et al. A study on the subhealth status of women by Del phi method [J]. China Maternal and Child Health Care! 2011! 26 (13): 1941-1942.

[14] Zhang Bingxiu, Li Guangshu. Chinese medicine health care and treatment in sub-healthy state [J]. Anhui Clinical Journal of traditional Chinese Medicine 15 (3): 254.

[15] Zhao Rong. Clinical observation of acupuncture and moxibustion in the treatment of female sub - health status \&bra; J \&ket. Shaanxi Traditional Chinese Medicine, 2011, 32 ( 3 ): 330-331.

[16] Lun Shifang, Pang Jun, Lei Longming, et al. Effects of massage therapy on psychosomatic symptoms in subhealthy population [J]. China Clinical Rehabilitation 9 (40): 100-101. 